\title{
Effects of probiotics and prebiotics on blood lipids
}

\author{
Gary R. J. Taylor and Christine M. Williams* \\ Hugh Sinclair Unit of Human Nutrition, Department of Food Science and Technology, University of Reading, \\ Reading RG6 6AP, UK
}

\begin{abstract}
Since the early work of Mann and Spoerry, probiotics in the form of fermented milk products have been reputed to have cholesterol-lowering properties in humans. However, studies conducted since the early 1970s have produced equivocal findings, with interpretation of the outcomes complicated by use of excessive quantities of product, inadequate sample sizes, failure to control nutrient intake and energy expenditure and variations in baseline blood lipids. More recent studies are of better quality, but fail to provide convincing evidence that 'live' fermented milk products have cholesterol-lowering efficacy in man. Future studies using probiotics should ensure adequate sample sizes sufficient to detect relatively small changes in blood cholesterol and should be conducted over longer periods of time. The recent introduction of the concept of prebiotics has directed attention towards the possibility that alterations in gut microflora induced by the fermentation of non-digestible components of the diet may also have the potential to influence systemic lipid metabolism. This possibility has been strengthened by the observation that in animals, dietary oligofructosaccharides cause suppression of hepatic triglyceride and VLDL synthesis, resulting in marked reductions in triglyceride, and to a lesser extent cholesterol, levels. Evidence for similar effects in humans is sparse and more studies are needed, particularly with respect to effects on postprandial triglyceride concentrations.
\end{abstract}

\section{Probiotics: Blood lipids: Prebiotics}

For over 20 years the primary focus of public health strategies aimed at reducing risk of $\mathrm{CHD}$ has been the attempt to reduce circulating blood cholesterol concentrations as a means of attenuating the primary pathological event of $\mathrm{CHD}$, atherosclerosis. Although there has been much recent debate concerning the mechanism(s) by which abnormal amounts and types of blood lipoproteins lead to increased risk of CHD (Austin et al. 1988), recent primary and secondary drug intervention trials unequivocally demonstrate that drug-induced reductions in serum cholesterol concentrations, even in men without existing disease and in those with only moderately raised levels, results in significant and marked reductions in mortality from heart disease (Scandinavian Simvastatin Survival Study Group, 1994; Levine et al. 1995; Shepherd et al. 1995). Since over $30 \%$ of the UK adult population have moderately elevated cholesterol levels, it is clear that effective dietary strategies for reducing population cholesterol levels would have considerable benefits in terms of reduced morbidity and mortality from CHD. In 1994 Law estimated that a $10 \%$ reduction in adult cholesterol levels (much less than is achieved using powerful cholestreol-lowering drugs) would result in a $10-25 \%$ reduction in the UK CHD mortality rate (Law et al. 1994). The need for a dietary rather than a drug approach to this problem is necessitated by the very large numbers of individuals affected which precludes the use of expensive drugs which may also carry risk of side effects. Current dietary strategies for prevention of CHD advocate adherence to low-fat/low-saturated fat diets (Department of Health, 1994). Although there is no doubt that under experimental conditions, low-fat diets offer an effective means of reducing blood cholesterol concentrations, on a population basis they appear to be much less effective, largely due to poor long-term compliance attributed to the low palatability and acceptability of these diets to the consumer. Not surprisingly, poor consumer acceptability of low-fat diets has lead to attempts to identify other dietary components which can reduce blood cholesterol levels, including the use of soluble fibres, soy protein, plants sterols and probiotics (usually live fermented milk products). More recently, evidence from animal studies has also suggested that prebiotics can produce moderate reductions in plasma cholesterol, but also very marked reductions in fasting and postprandial blood triglycerides. These effects, not yet observed in human subjects, are of particular interest in the light of increasing evidence for pro-atherogenic and prothrombotic effects of raised blood triglyceride levels (Havel, 1994). The aim of this review is 
to consider the nature and quality of the evidence for cholesterol-lowering effects of probiotics in humans and to consider the theoretical basis for effects of prebiotics on blood lipids in animals and humans.

\section{Probiotics and blood lipids}

Since 1974 when Mann and Spoerry showed an $18 \%$ fall in plasma cholesterol after feeding 4-5 litres of fermented milk per day for 3 weeks to Maasai warriors (Mann \& Spoerry, 1974), there has been considerable interest in the effect of probiotics on human lipid metabolism. Although this study is frequently quoted as the basis for much of the animal and human work subsequently carried out in the 1970s and 1980s, consideration of the details of the study show a number of problems which shed doubt on the interpretation of the findings. The investigators original intention was to feed 4-5 litres of pasteurized European milk, fermented with a wild culture of lactobacillus, to twenty-four male Maasai warriors, for $6 \mathrm{~d}$ out of each week. However, demand rose to approximately $8.3 \mathrm{l} / \mathrm{d}$ $(5500 \mathrm{kcal} / \mathrm{d})$ and an exercise programme was started to prevent weight gain. Eight subjects feigned injury to avoid this exercise and thus gained substantial weight. After 3 weeks the study was abandoned due to the inability to control the food intake. In eight subjects who gained $>5 \mathrm{lbs}$ body weight the mean serum cholesterol fell by $0.73 \mathrm{mmol} / \mathrm{l}(18.2 \%)$. The total group mean serum cholesterol also decreased significantly (Table 1). Although the observation of marked reductions in cholesterol despite increased body weight is surprising, the results of this trial are now simply a curiosity due to the confounding effect of introducing an exercise regime during the trial and the inability to control food intake which casts doubt over the causality of the findings. As a follow-up to the Maasai study Mann (1977), fed small groups of US volunteers 4 litres of yoghurt per day (microbiological activity unspecified) over 12 days and reported a $37 \%$ fall in serum cholesterol (tabulated data in the report indicated only a $16.8 \%$ fall in cholesterol). When intake was reduced to $2 \mathrm{l} / \mathrm{d}$ the cholesterol-lowering effect was maintained but 1 litre failed to maintain the lower cholesterol values and levels returned to baseline values within 2 weeks. The rate of cholesterol biosynthesis was monitored in this study by measuring the specific activity of plasma digitoninprecipitated sterols, $2 \mathrm{~h}$ after a pulse of ${ }^{14} \mathrm{C}$ acetate. A $28 \%$ fall in acetate incorporation was reported by day 16 , after 12 days of 4 litres of yoghurt per day. Mann proposed the presence of a 'milk factor' to explain the fall in serum cholesterol and suggested this was hydroxymethyl glutarate, a 3HMG-CoA reductase inhibitor (Mann, 1997). A surprising aspect of this study is that habitual diet, body weight and daily activity levels were maintained constant despite the fact that subjects consumed over 4 litres of yoghurt per day providing approximately $2640 \mathrm{kcal}$ of additional energy intake.

The human studies investigating the potential lipidlowering effect of yoghurts published since the early studies of Mann and co-workers have varied in the quality of study design and the type of yoghurt used. Some studies have observed significant reductions in total plasma cholesterol levels (Howard \& Marks. 1979; Hepner et al. 1979; Jaspers et al. 1984; Agerbaek et al. 1995; Richelsen et al. 1996) whereas others have been negative (Rossouw et al. 1981; Thompson et al. 1982; Massay, 1984; McNamara et al. 1989) (Table 1). One of the major criticisms of the studies of Mann and co-workers were the very large volumes of product employed and the lack of suitable control or placebo groups. To avoid the drastic alteration of the dietary intake encountered in the early studies, Hepner devised a regime

Table 1. Details of human studies to evaluate the cholesterol-lowering properties of fermented milk products

\begin{tabular}{|c|c|c|c|c|c|c|}
\hline Author & Subjects $(n)$ & Product (vol/type) & Duration & Total cholesterol & Significance & LDL cholesterol \\
\hline Mann et al. (1974) & $24 M$ & 8.31 lacto yoghurt & 3 weeks & $-9.6 \%$ & $P<0.001$ & NA \\
\hline \multirow[t]{2}{*}{ Mann (1977) } & $3 \mathrm{M} 1 \mathrm{~F}$ & $4 \mid$ WMY & 12 days & $-16.8 \%$ & $P<0.05$ & NA \\
\hline & $3 \mathrm{M} 2 \mathrm{~F}$ & 2 I SMY & 12 days & $-23.2 \%$ & $P<0.05$ & NA \\
\hline Howard et al. (1979) & 10 & 31 & 3 weeks & $-5.5 \%$ & $P<0.05$ & NA \\
\hline \multirow[t]{2}{*}{ Hepner et al. (1979) } & $6 \mathrm{M} 4 \mathrm{~F}$ & $720 \mathrm{ML}(\mathrm{A})$ & 4 weeks & $-5.4 \%$ & $P<0.01$ & NA \\
\hline & $5 \mathrm{M} 3 \mathrm{~F}$ & $720 \mathrm{ml}(\mathrm{B})$ & 4 weeks & $-8.9 \%$ & $P<0.01$ & NA \\
\hline Rossouw et al. (1981) & $11 M$ & 21 yoghurt & 3 weeks & $+16 \%$ & $P<0.01$ & $+12 \% P<0.001$ \\
\hline Thompson et al. (1982) & 13 & 1 I UPY & 3 weeks & NS & NS & NS \\
\hline Bazzare et al. (1983) & $5 M 16 F$ & $750 \mathrm{~g}$ yoghurt & 1 week & $-8.7 \%$ & $?$ & NA \\
\hline Massay (1984) & $30 \mathrm{~F}$ & $480 \mathrm{ml}$ yoghurt & 4 weeks & NS & NS & NS \\
\hline Jaspers et al. (1984) & $10 \mathrm{M}$ & $681 \mathrm{~g}$ yoghurt & 2 weeks & $-11.64 \%$ transient & $P<0.05$ & NS \\
\hline McNamara et al. (1989) & $18 M$ & 16 oz LFY & 4 weeks & NS & NS & NS \\
\hline Agerbaek et al. (1995) & $58 \mathrm{M}$ & $200 \mathrm{ml}$ UPY & 6 weeks & $-6.1 \%$ & $P<0.001$ & $-9.8 \% P<0001$ \\
\hline Richelsen et al. (1996) & $47 \mathrm{M} \mathrm{43W}$ & $200 \mathrm{ml}$ UPY & 24 weeks & NA & NA & $\begin{array}{c}-9 \% \text { transient } \\
P<0.05\end{array}$ \\
\hline Sessions et al. (1997) & $78 \mathrm{M} 76 \mathrm{M}$ & $200 \mathrm{ml}$ UPY & 12 weeks & NS & NS & NS \\
\hline
\end{tabular}

F, female; M, male; WMY, whole milk yoghurt; UPY, unpasteurized yoghurt; SMY, skimmed milk yoghurts; PY, pasteurized yoghurt; LFY, low fat yoghurt; NA, data not available. 
imposing a $720 \mathrm{ml}$ test dose of yoghurt for 4 weeks controlled with $720 \mathrm{ml}$ of milk in a cross-over study, with 4 weeks of normal diet between the two study periods (Hepner et al. 1979). The design of the study was rigorous but equivocal findings were observed, despite the fact that this represents one of the more positive studies. A significant fall in serum cholesterol occurred in both the yoghurt and milk groups after 1 week during the first arm and in the yoghurt group only in the first week of the second arm. The authors concluded that although the cholesterol-lowering effects of the yoghurt were greater than the milk, there may also have been a cholesterol-lowering effect of the milk supplement. An alternative explanation is that during the first week of the first arm, all of the subjects were 'adapting' to being on an experimental study. It has been shown by Rossouw et al. (1979) that participating for a single week in a 'nutritional trial' without introducing any dietary alteration resulted in a reduction in plasma total cholesterol of $7 \%$. This effect is probably due to an alteration of lifestyle that may be conscious, but is more likely to be a subconscious heightening of the awareness of food intake and diet, especially if during this period dietary records are being completed. Of the early negative studies that have been published, those of Thompson et al. (1982), Massay (1984) and McNamara et al. (1989) incorporated a run-in period. The study of McNamara et al. (1989) was one of the more carefully designed studies with particular emphasis placed on the run-in period. A period of 3 weeks on a baseline low-fat/low-cholesterol diet preceded the 4-week trial period of baseline diet plus low-fat yoghurt which was followed by a 4-week placebo period of baseline diet and a non-fermented milk concentrate to mimic the composition of unfermented yoghurt. The eighteen subjects were normolipidaemic males who were counselled to maintain normal work/stress patterns, meal times and smoking, alcohol and exercise habits. Dietary intake and body weight remained constant and there was no significant change in serum cholesterol, LDL, HDL or triglycerides for any of the three periods - baseline run-in, yoghurt or placebo. In contrast most of the early studies that showed a cholesterol-lowering effect of probiotics, such as Hepner et al. (1979) and Jaspers et al. (1984), were designed without yoghurt-free adjustment periods, or a run-in period prior to the test period. Thus the failure to use a run-in period may be responsible for some of the apparently positive findings of some of these studies.

The study of Rossouw et al. (1979) also reports on the overall nutritional consequences of adding large amounts of milk products ( 2 litres of either unspecified yoghurt, whole milk or skimmed milk product) to the diets of normal healthy subjects. During the experimental 3 weeks, energy intake was not significantly different between groups or compared with baseline values, but energy intake in the skimmed milk group tended to be lower than in the other groups. All groups gained weight $(0.7-1.5 \mathrm{~kg})$ and showed higher intakes of protein $(+40 \mathrm{~g} / \mathrm{d})$, calcium $(2 \times)$, vitamin $A$ $(+1000 \mathrm{IU} /$ day) and cholesterol. In the whole milk and yoghurt groups, daily cholesterol intake increased to $800-$ $900 \mathrm{mg}$ and $400-500 \mathrm{mg}$, respectively, from baseline intakes of $300-500 \mathrm{mg}$. In this study consumption of yoghurt for 3 weeks prompted a $16 \%$ increase in blood cholesterol after 2 weeks which subsequently returned to the baseline value at 3 weeks. A similar response was seen in the whole milk group but not in the skimmed milk group in whom cholesterol levels were reduced over the study period. It is likely that similar confounding dietary changes have occurred in other trials but these have not been monitored, nor commented upon.

Given that intra-individual variation in blood cholesterol over a few months ranges from 5 to $15 \%$ and that even in the best laboratories the analytical precision of the cholesterol assay is $2-7 \%$, it is clear that any study aimed at detecting small $(5-15 \%)$ changes in blood cholesterol will require large sample sizes to ensure sufficient statistical power. Given these considerations, none of the studies described above have sufficient statistical power to detect changes in cholesterol of $<15 \%$, yet it is clear that if probiotics do have a cholesterol-lowering effect it is a relatively weak one compared with the effect of the powerful cholesterol-lowering drugs. For this reason studies need to be designed with larger numbers of subjects, where the effects of confounding dietary changes are minimized and in which the product is consumed over a sufficient period of time to ensure a sustained response. Some of the more recent studies have used larger sample sizes thereby increasing the possibility of obtaining positive results if the product is effective.

Following unpublished observations of an animal study which showed that a yoghurt containing $E$. faecium was hypocholesterolaemic. Agerbaek et al. (1995) tested $200 \mathrm{ml}$ daily of this live yoghurt in a parallel design against an identical but chemically fermented product, for 6 weeks in fifty-eight middle-aged men with moderately raised cholesterol levels $(5.0-6.5 \mathrm{mM})$. They observed a $9.8 \%$ reduction in LDL cholesterol $(P<0.001)$ in the live yoghurt group, which was sustained over the period of 6 weeks (Table 1). This was a well-controlled trial which excluded many variables such as age, sex, body weight and baseline cholesterol and which minimized the effect of variables such as diet, smoking, exercise and alcohol consumption. However, precautions against a skew in the randomization process had not been foreseen and both the baseline LDL and plasma total cholesterol levels were significantly higher in the test group compared with the controls. The fall in these parameters seen in the test group could therefore be ascribed to regression towards the mean. In 1996, another long-term study (6 months), using the same product and employing a similar design, was carried out in eighty-seven males and females aged 50-70 years (Richelsen et al. 1996). This study produced some equivocal data that were partly explained through a fall in the titre of the active product at 12 weeks. At 12 weeks, a significant reduction in LDL cholesterol was observed in the live yoghurt group but this was not fully sustained and values increased in the second 3 months of the trial. Since there was also a small reduction in the LDL cholesterol levels in the control group, cholesterol values were not significantly different between the groups at the end of the trial.

More recently a similar trial of the same product containing $E$. faecium was conducted in 160 middle-aged men and women with moderately raised cholesterol (Sessions et al. 1997). Subjects were recruited from three 
centres in southern England and the Midlands and consumed $200 \mathrm{ml}$ daily of either the live yoghurt or a chemically fermented placebo in a parallel design for 12 weeks. Stratified randomization was employed to ensure the groups were comparable for age, sex, BMI and baseline fasting cholesterol levels. Subjects were instructed not to change their dietary habits during the trial and dietary assessment confirmed adherence to this instruction. The titre of the product was maintained throughout the trial. During a 2-week run-in period, subjects in both the placebo and live yoghurt groups showed significant reductions in blood cholesterol levels but thereafter there were no further changes in either of the groups and no significant differences between groups at any of the time points. These data are consistent with the conclusions drawn by Rossouw et al. (1981) which indicate that apparent effects of probiotics on blood cholesterol levels may be attributed to the reductions in blood lipids observed in subjects at the beginning of intervention studies which are difficult to prevent and which appear to be due to conscious or subconscious changes in dietary and other lifestyle habits.

\section{Prebiotics and human lipid metabolism}

The possibility that dietary-induced alterations in gut microflora may have beneficial effects on human lipid metabolism is not a new concept but has been lent greater impetus by recent work showing specific effects of certain nondigestible carbohydrates on the gut bifidobacteria (Gibson et al. 1995) and by animal studies which show marked effects of dietary oligofructosaccharides both in stimulating bifidobacteria and in reducing serum triglyceride concentrations (Delzenne et al. 1993; Delzenne \& Roberfroid, 1994; Gibson \& Roberfroid, 1994; Fiordaliso et al. 1995). The term prebiotic has been coined to describe these dietary carbohydrates which are able to stimulate, specifically, the growth of potentially beneficial bacteria at the expense of the more harmful pathogenic micro-organisms (Gibson \& Roberfroid, 1994). The possibility that prebiotics may have beneficial effects on blood lipids has attracted considerable attention because, unlike the probiotics, prebiotics are not subject to the difficulties faced by the survival of an ingested micro-organism. Prebiotics also have greater possibilities for incorporation into a wide range of common foodstuffs and do not have limited shelf lives. However, as yet, studies of their beneficial effects on blood lipid levels in man are limited so that evidence for this particular health benefit is largely provided by a number of convincing animal studies.

In Wistar rats oligofructose as $10 \%$ inulin reduces postprandial serum triglycerides after 5 weeks to between 30 and $40 \%$ of control values, reduces hepatic triglyceride content by $20 \%$ and markedly reduces the activity and gene expression of fatty acid synthase, the rate-limiting enzyme of fatty acid synthesis (Delzenne et al. 1993; Delzenne \& Roberfroid, 1994; Fiordaliso et al. 1995; Kok et al. 1996a,b) A fibre-free diet supplemented with $10 \%$ oligofructose results in the greatest reduction in serum triglycerides and also a small reduction in serum cholesterol concentration which appears to be secondary to reduced hepatic VLDL secretion (Delzenne \& Roberfroid, 1994).

However, human volunteers given oligofructose at a dose of $20 \mathrm{~g} / \mathrm{d}$ for 4 weeks - which has been shown to produce alteration in gut bifidobacteria (Gibson et al. 1995) showed no change in fasting serum triglycerides or cholesterol concentrations (Luo et al. 1996). Yamashita et al. (1984) showed a small reduction in LDL cholesterol in eighteen diabetics taking $8 \mathrm{~g} / \mathrm{d}$ of oligofructose for $14 \mathrm{~d}$ but no change in serum triglycerides. Similarly no effects of feeding dietary oligofructosaccharides on serum insulin and glucose levels were observed in normal healthy subjects (Rumessen et al. 1990). A recent human study fed $9 \mathrm{~g} / \mathrm{d}$ of inulin to twelve volunteers for 1 month as part of a breakfast cereal. Fasting serum triglycerides were reduced by $27 \%$ (Table 2) but cholesterol was reduced by only $5 \%$, again suggesting the major effect was likely to be on the VLDL, as is suggested in the animal studies. Analysis of the gut microflora showed no change after this regime, but breath hydrogen was significantly elevated during the inulin-fed diet (Canzi et al. 1995). In contrast to the findings of Canzi et al. (1995), Pedersen and co-workers found no effect on fasting total, LDL or HDL cholesterol or serum triglycerides of $14 \mathrm{~g} / \mathrm{d}$ of inulin incorporated into a low-fat spread for a period of 4 weeks. This double-blind randomized trial which was conducted in a group of sixty-six healthy women suggests that if lipid-lowering effects of inulin are to be observed in humans larger doses or longer periods of feeding may be required.

Consideration of the metabolic response to prebiotics is beyond the scope of this review; however, the assumption that effects of non-digestible carbohydrates on systemic lipid metabolism are solely mediated through effects of bacterial fermentation products may be incorrect, and other possibilities, including alterations in gut hormones and effects of other by-products of bacterial metabolism are also currently under investigation.

\section{Discussion}

Data from studies conducted since the early 1970s indicate that the role of fermented milk products as hypocholesterolaemic agents in humans is equivocal. There are many limitations in the study designs with early studies using

Table 2. Human studies investigating the effects of oligofructosaccharides on fasting triglyceride (TAG) levels

\begin{tabular}{lllcccc}
\hline Author & Date & Prebiotic & Dose $(\mathrm{g} / \mathrm{d})$ & Duration & Sample size $(n)$ & TAG \\
\hline Yamashita & 1984 & FOS & 8 & 14 days & 18 & NS \\
Luo et al. & 1996 & FOS & 20 & 4 weeks & 12 & NS \\
Canzi et al. & 1995 & Inulin & 9 & 4 weeks & 12 & $-27 \%$ \\
Pedersen et al. & 1997 & Inulin & 14 & 4 weeks & 66 & NS \\
\hline
\end{tabular}


excessive volumes of yoghurt and failing to control background dietary intake and physical activity levels. Many of the studies have not used run-in periods to stabilize baseline measurements and have used infrequent sampling periods. Significant changes in blood lipids in control groups is a frequent observation and may reflect random variation in cholesterol levels, changes in diet or effects of the placebo product themselves. The major limitation of the studies is their small sample size and any future work in this area should be conducted following estimation of sample size with realistic estimates of likely response taking into account known variations in blood cholesterol levels over time. Although beyond the scope of this review, further consideration should also be given to the mechanisms underlying the putative cholesterol-lowering effects of probiotics, the problems of survivability in the large bowel and the possible role of prebiotics in maintaining adequate survival and colonization of appropriate cholesterol-lowering species. Data concerning effects of dietary prebiotics on blood lipids in humans are sparse and that which is available is contradictory. In the light of the known day-to-day variations in fasting triglyceride concentrations and increasing evidence for harmful effects of elevated postprandial, as opposed to fasting, triglycrides (Groot et al. 1991; Patsch, 1992; Havel, 1994; Hokanson \& Austin, 1996) more information is needed on the effects on postprandial triglycerides, of adding inulin and other putative prebiotics to the diet. As in the case of the probiotics, lack of credibility in this area partly relates to the common, but mistaken, assumption that blood lipids are highly repeatable measures both within and between individuals and that response to a single dietary intervention can be readily demonstrated using small numbers of subjects. Further studies are needed using a wider range of dose levels and food vehicles. Most importantly, studies should include realistic numbers of subjects and multiple time point, including baseline, measurements should be made.

\section{References}

Agerbaek M, Gerdes LU \& Richelsen B (1995) Hypocholesterolaemic effect of a new fermented milk product in healthy middle-aged men. European Journal Clinical Nutrition 49, 346-352.

Austin MA, Breslow JL, Hennekens CH, Buring JE, Willett WC \& Krause RM (1988) Low density lipoprotein subclass patterns and risk of myocardial infarction. Journal of American Medical Association 260, 1917-1921.

Bazarre TL, Wu SL \& Yuhas JA (1983) Total and HDLcholesterol concentration following yoghurt and calcium supplementation. Nutrition Reports International 28, 1225-1232.

Canzi E, Brighenti F, Casiraghi MC, Del Puppo E \& Ferrari A (1995) Prolonged consumption of inulin in ready to eat breakfast cereals, effects on intestinal ecosystem, bowel habits and lipid metabolism. Proceedings of the COST 92 Conference 'Dietary fibre and fermentation in the colon'. Helsinki.

Delzenne NM, Kok N, Fiordaliso MF, Deboyser DM, Goethels FM \& Roberfroid MB (1993) Dietary fructooligosaccharides modify lipid metabolism in rats. American Journal of Clinical Nutrition 57, 820 S.

Delzenne NM \& Roberfroid MR (1994) Physiological effects of non-digestible oligosaccharides. Lebensmittel Wissenschaft und Technologie 27, 1-6.
Department of Health (1994) Nutritional Aspects of Cardiovascular Disease. Report on Health and Social Subjects 46. London: HMSO.

Fiordaliso MF, Kok N, Desager JP, Goethels F, Deboyser D, Roberfroid M \& Delzenne N (1995) Oligofructose supplemented diet lowers serum and VLDL concentrations of triglycerides, phospholipids and cholesterol in rats. Lipids 30, 163-167.

Gibson GR, Beatty ER, Wang X \& Cummings JH (1995) Selective stimulation of bifidobacteria in the human colon by oligofructose and inulin. Gastroenterology 108, 975-982.

Gibson GR \& Roberfroid MB (1994) Dietary modulation of the human colonic microbiota. Introducing the concept of prebiotics. Journal of Nutrition 125, 1401-1412.

Groot PHE, Van Stiphout WAHJ, Krauss XH, Jansen H, Van Tol A, Van Ramshorst E, Chin-On S, Hofman A, Cresswell SR \& Havekes L (1991) Postprandial lipoprotein metabolism in normolipidaemic men with and without coronary artery disease. Arteriosclerosis and Thrombosis 11, 653-662.

Havel R (1994) McCollum Award Lecture 1993, Triglyceride-rich lipoproteins and atherosclerosis - a new perspective. American Journal Clinical Nutrition 59, 795-799.

Hepner G, Fried R, Jeor SS, Fusetti L \& Morin R (1979) Hypocholesterolaemic effect of yoghurt and milk. American Journal Clinical Nutrition 32, 19-24.

Hokanson JE \& Austin MA (1996) Plasma triglyceride level is a risk factor for cardiovascular disease independent of highdensity lipoprotein cholesterol level, a meta-analysis of population-based prospective studies. Journal of Cardiovascular Risk 3, 213-219.

Howard AN \& Marks J (1979) Effect of mil products on serum cholesterol. Lancet ii 957.

Jaspers DA, Massey LK \& Luedecke LO (1984) Effect of consuming yoghurts prepared with three culture strains on human serum lipoproteins. Journal of Food Science 49, 1178-1181.

Kok N, Roberfroid M, Robert A \& Delzenne N (1996a) Involvement of lipogenesis in the lower VLDL secretion induced by oligofructose in rats. British Journal of Nutrition 76, 880-891.

Kok N, Roberfroid M \& Delzenne N (1996b) Dietary oligofructose modifies the impact of fructose on hepatic triacylglycerol metabolism. Metabolism 45, 1547-1550.

Law MR, Wald NJ \& Hackshaw A (1994) Systematic underestimation of association between serum cholesterol concentration and ischaemic heart disease in observational studies, data from the BUPA study. British Medical Journal 308, 363-366.

Levine GN, Keaney JF \& Vita JA (1995) Cholesterol reduction in cardiovascular disease. Clinical benefits and possible mechanisms. New England Journal Medicine 332, 512-521.

Luo J, Rizkalla SW, Alamowiteh C, Boussairi A, Blayo A, Barry J-L, Laffitte A, Guyon F, Bornet FRJ \& Slama G (1996) Chronic consumption of short-chain fructooligosaccharides by healthy subjects decreased basal hepatic glucose production but had no effect on insulin-stimulated glucose metabolism. American Journal Clinical Nutrition 63, 939-945.

McNamara DJ, Lowell AE \& Sabb JE (1989) Effect of yoghurt intake on plasma lipid and lipoprotein levels in normolipidemic males. Atherosclerosis 79, 167-171.

Mann GV (1977) A factor in yoghurt which lowers cholesteremia in man. Atherosclerosis 26, 335-340.

Mann GV \& Spoerry A (1974) Studies of a surfactant and cholesteremia in the Maasai. American Joumal Clinical Nutrition 27, 464-469.

Massay LK (1984) Effect of changing milk and yoghurt consumption on human nutrient intake and serum lipoproteins. Journal Dairy Science 67, 255-262.

Patsch W (1992) Relation of triglyceride metabolism and coronary artery disease. Arteriosclerosis and Thrombosis 12, 13361345. 
Pedersen A, Sandstrom B \& Van Amelsvoort JMM (1997) The effect of ingestion of inulin on blood lipids and gastrointestinal symptoms in healthy females. British Journal of Nutrition $\mathbf{7 8}$, 215-222.

Richelsen B, Kristensen K \& Pedersen SB (1996) Long-term (6 months) effect of a new fermented milk product on the level of plasma lipoproteins - a placebo-controlled and double blind study. European Journal of Clinical Nutrition 50, $811-815$

Rossouw JE, Burger EM, Van der Vyver P \& Ferreira JJ (1981) The effect of skim milk, yoghurt and full cream milk on human serum lipids. American Journal Clinical Nutrition 34, 351-356.

Rumessen JJ, Bode S, Hamberg O \& Gudmand-Hoyer E (1990) Fructans of Jerusalem artichokes, intestinal transport, absorption, fermentation and influence on blood glucose, insulin and C-peptide responses in healthy subjects. American Journal Clinical Nutrition 52, 675-681.

Scandinavian Simvastatin Survival Study Group (1994) Randomised trial of cholesterol lowering in 4444 patients with coronary heart disease. Lancet 344, 1383-1389.

Sessions VA, Lovegrove JA, Taylor GRJ, Dean TS, Williams CM, Sanders TAB, Macdonald I \& Salter A (1997) The effects of a new fermented milk product on total plasma cholesterol, LDL cholesterol and apolipoprotein B concentrations in middle aged men and women. Proceedings of the Nutrition Society 56, 120A.

Shepherd J, Cobbe SM, Ford I, Isles CG, Lorimer AR, Macfarlane PW, McKillop JH \& Packard CJ (1995) Prevention of coronary heart disease with pravastatin in men with hypercholesterolaemia. New England Journal of Medicine 333, 1301-7.

Thompson LU, Jenkins DJA, Amer V, Reichert R, Jenkins A \& Kamulsky J (1982) The effect of fermented and unfermented milks on serum cholesterol. American Journal Clinical Nutrition 36, 1106-1111.

Yamashita K, Kawai K \& Itakura M (1984) Effects of fructooligosaccharides on blood glucose and serum lipids in diabetic subjects. Nutrition Research 4, 961-966. 